Kyushu J. Math.

Vol. 58, 2004, pp. 353-371

\title{
ON THE FIRST SUBCONSTITUENT OF A QUADRATIC FORMS SCHEME
}

\author{
Seiichi IWAMOTO \\ (Received 20 October 2003)
}

\begin{abstract}
As a step to find the eigenvalues of the first subconstituent $X_{1}$ of a quadratic forms scheme, we determine the orbits of $G L_{n}(q)$ acting on $X_{1} \times X_{1}$.
\end{abstract}

\section{Introduction}

Let $q$ be a power of an odd prime and let $G F(q)$ denote the finite field of $q$ elements. Let $n$ be a natural number $(n \geq 3)$ and let $X$ denote the set of symmetric matrices over $G F(q)$ of size $n$. For an integer $i(0 \leq i \leq(n+1) / 2)$, let $X_{i}$ denote the subset of $X$ that consists of matrices in $X$ of rank $2 i-1$ or $2 i$. Let $R_{i}(0 \leq i \leq$ $(n+1) / 2)$ be a relation on $X$ defined by $\left(M, M^{\prime}\right) \in R_{i} \Longleftrightarrow M^{\prime}-M \in X_{i}$, i.e. $\operatorname{rank}\left(M^{\prime}-M\right)=2 i-1,2 i$. Then we have a $P$ - and $Q$-polynomial association scheme $\mathcal{X}=\left(X,\left\{R_{i}\right\}_{0 \leq i \leq(n+1) / 2}\right)$, which is called the quadratic forms scheme [1]. The subset $X_{i}$ is called the $i$ th subconstituent.

Let $\Gamma$ denote the graph whose vertex set is $X_{1}$ and whose edge set is the restriction of $R_{1}$ on $X_{1} \times X_{1}$. In the problem of characterizing the quadratic forms scheme by the parameters, the eigenvalues of the graph $\Gamma$ on the first subconstituent $X_{1}$ are crucially important in the sense that certain subsets of them are in one-to-one correspondence with the isomorphism classes of irreducible thin $T$-modules of endpoint 1 , where $T$ is the Terwilliger algebra of the $P$ - and $Q$-polynomial association scheme, and that they determine the global structure of the $P$ - and $Q$-polynomial association scheme to some extent [4]. Note that the eigenvalues of the first subconstituent graph $\Gamma$ are known for other classical forms schemes, i.e. bilinear, hermitian and alternating forms schemes, whereas those for the quadratic forms scheme are yet to be found [3].

Let $G$ denote the general linear group $G L_{n}(q)$, the group of non-singular matrices over $G F(q)$ of size $n$. Then $G$ acts on $\Gamma$ as a group of automorphisms $M^{g}={ }^{\mathrm{t}} g M g$ for $g \in G, M \in X_{1}$. Let $G$ act on $X_{1} \times X_{1}$ by $\left(M, M^{\prime}\right)^{g}=\left(M^{g}, M^{\prime g}\right)$ for $g \in G, M, M^{\prime} \in X_{1}$. Each of the $G$-orbits on $X_{1} \times X_{1}$ gives rise to a relation 
on $X_{1} \times X_{1}$ and we have a coherent configuration on $X_{1}$ with the $G$-orbits on $X_{1} \times X_{1}$ as the defining relations [2]. Note that the coherent configuration is not an association scheme, since $G$ is not transitive on $X_{1}$. The graph $\Gamma$ is reconstructed from the coherent configuration by fusing relations, and the eigenvalues of $\Gamma$ can be obtained in the framework of the coherent configuration by determining the intersection numbers $p_{i j}^{k}$ and the eigenvalues of the intersection matrix $B_{i}=\left(p_{i j}^{k}\right)$. In this paper, we determine the $G$-orbits on $X_{1} \times X_{1}$ as the first step of our approach to the eigenvalue problem of $\Gamma$. We plan to determine the eigenvalues of $\Gamma$ in the framework of the coherent configuration in a forthcoming paper. Throughout this paper, we keep the notation fixed as in this section.

\section{The $G$-orbits on $X_{i}$}

Let $\epsilon$ be a fixed non-square element in $G F(q)$. $G$ acts on $X_{i}$ by $M^{g}={ }^{\mathrm{t}} g M g$ ( $g \in G, M \in X_{i}$ ) and it is well known that $G$ has four orbits on $X_{i}$ represented by

$$
\left(\begin{array}{cc}
J_{2 i-1}^{+} & \mathbf{0} \\
\mathbf{0} & \mathbf{0}
\end{array}\right),\left(\begin{array}{cc}
J_{2 i-1}^{-} & \mathbf{0} \\
\mathbf{0} & \mathbf{0}
\end{array}\right),\left(\begin{array}{cc}
J_{2 i}^{+} & \mathbf{0} \\
\mathbf{0} & \mathbf{0}
\end{array}\right),\left(\begin{array}{cc}
J_{2 i}^{-} & \mathbf{0} \\
\mathbf{0} & \mathbf{0}
\end{array}\right),
$$

respectively, where

$$
\begin{aligned}
J_{2 i-1}^{+} & =\left(\begin{array}{cc|c}
\mathbf{0} & I_{i-1} & 0 \\
I_{i-1} & \mathbf{0} & 0 \\
\hline 0 & 0 & 1
\end{array}\right) \quad(\text { size } 2 i-1), \\
J_{2 i-1}^{-} & =\left(\begin{array}{cc|c}
\mathbf{0} & I_{i-1} & 0 \\
I_{i-1} & \mathbf{0} & 0 \\
\hline 0 & 0 & \epsilon
\end{array}\right) \quad(\text { size } 2 i-1), \\
J_{2 i}^{+} & =\left(\begin{array}{cc}
\mathbf{0} & I_{i} \\
I_{i} & \mathbf{0}
\end{array}\right) \\
J_{2 i}^{-} & =\left(\begin{array}{cc|cc}
\mathbf{0} & I_{i-1} & 0 & 0 \\
I_{i-1} & \mathbf{0} & 0 & 0 \\
\hline 0 & 0 & 1 & 0 \\
0 & 0 & 0 & -\epsilon
\end{array}\right) \quad(\text { size } 2 i),
\end{aligned}
$$

with $I_{j}$ the identity matrix of size $j$ and $\mathbf{0}$ the zero matrix of the corresponding size. 
Let $J$ be one of $J_{2 i-1}^{+}, J_{2 i-1}^{-}, J_{2 i}^{+}, J_{2 i}^{-}$and set

$$
O_{m}(q, J)=\left\{g \in G L_{m}(q) \mid{ }^{\mathrm{t}} g J g=J\right\}
$$

where $m$ is the matrix size of $J$. For natural numbers $k, l$, let $\mathcal{M}_{k, l}(q)$ denote the set of $k \times l$ matrices over $G F(q)$. Then the stabilizer of

$$
\begin{gathered}
\left(\begin{array}{ll}
J & 0 \\
0 & 0
\end{array}\right) \in X_{i} \text { in } G \text { is } \\
G_{J}=\left\{g \in G \mid g=\left(\begin{array}{ll}
A & 0 \\
B & C
\end{array}\right), \begin{array}{l}
A \in O_{m}(q, J), \quad B \in \mathcal{M}_{n-m, m}(q), \\
C \in G L_{n-m}(q)
\end{array}\right\} .
\end{gathered}
$$

Let $X_{J}$ denote the $G$-orbit on $X_{i}$ represented by $\left(\begin{array}{cc}J & 0 \\ 0 & 0\end{array}\right)$. Then $G_{J} \backslash G$, the set of cosets of $G$ by $G_{J}$, is isomorphic to $X_{J}$ as $G$-sets by the correspondence

$$
G_{J} \cdot g \mapsto{ }^{\mathrm{t}} g\left(\begin{array}{cc}
J & 0 \\
0 & 0
\end{array}\right) g
$$

Let $\mathcal{M}_{k, l}^{*}(q)$ denote the set of $k \times l$ matrices over $G F(q)$ of full rank, i.e. of rank $j$, where $j=\min \{k, l\}$. For $g \in G$, write $g$ as

$$
g=\left(\begin{array}{l}
M \\
N
\end{array}\right)
$$

with $M \in \mathcal{M}_{m, n}^{*}(q), N \in \mathcal{M}_{n-m, n}^{*}(q)$. Then we have

$$
{ }^{\mathrm{t}} g\left(\begin{array}{ll}
J & 0 \\
0 & 0
\end{array}\right) g={ }^{\mathrm{t}} M J M .
$$

LEMMA 2.1. For $g, g^{\prime} \in G$, write $g, g^{\prime}$ as

$$
g=\left(\begin{array}{l}
M \\
N
\end{array}\right), \quad g^{\prime}=\left(\begin{array}{l}
M^{\prime} \\
N^{\prime}
\end{array}\right)
$$

with $M, M^{\prime} \in \mathcal{M}_{m, n}^{*}(q), N, N^{\prime} \in \mathcal{M}_{n-m, n}^{*}(q)$. Then $g^{\prime}=h g$ for some $h \in G_{J}$ if and only if $M^{\prime}=A M$ for some $A \in O_{m}(q, J)$.

Proof. Suppose $g^{\prime}=h g$ for some $h \in G_{J}$. Then

$$
h=\left(\begin{array}{ll}
A & 0 \\
B & C
\end{array}\right)
$$


for some $A \in O_{m}(q, J), B \in \mathcal{M}_{n-m, m}(q), C \in G L_{n-m}(q)$. So,

$$
\left(\begin{array}{l}
M^{\prime} \\
N^{\prime}
\end{array}\right)=\left(\begin{array}{ll}
A & 0 \\
B & C
\end{array}\right)\left(\begin{array}{l}
M \\
N
\end{array}\right)
$$

and we have $M^{\prime}=A M$. Conversely, suppose that $M^{\prime}=A M$ for some $A \in O_{m}(q, J)$. Then

$$
{ }^{\mathrm{t}} g^{\prime}\left(\begin{array}{ll}
J & 0 \\
0 & 0
\end{array}\right) g^{\prime}={ }^{\mathrm{t}} M^{\prime} J M^{\prime}={ }^{\mathrm{t}} M J M={ }^{\mathrm{t}} g\left(\begin{array}{ll}
J & 0 \\
0 & 0
\end{array}\right) g,
$$

since ${ }^{\mathrm{t}} A J A=J$. So $g, g^{\prime}$ belong to the same $G_{J}$-coset.

The set $\mathcal{M}_{m, n}^{*}(q)$ admits the action of $O_{m}(q, J)$ from the left and that of $G$ from the right. Let $O_{m}(q, J) \backslash \mathcal{M}_{m, n}^{*}(q)$ denote the set of orbits of $O_{m}(q, J)$ on $\mathcal{M}_{m, n}^{*}(q)$. Then Lemma 2.1 states that the mapping $G_{J} \cdot g \mapsto O_{m}(q, J) M\left(g=\left(\begin{array}{c}M \\ N\end{array}\right)\right)$ from $G_{J} \backslash G$ to $O_{m}(q, J) \backslash \mathcal{M}_{m, n}^{*}(q)$ is an isomorphism of $G$-sets.

Thus we have the following lemma.

LEMMA 2.2. $O_{m}(q, J) \backslash \mathcal{M}_{m, n}^{*}(q)$ is isomorphic to $X_{J}$ as $G$-sets by the correspondence

$$
O_{m}(q, J) M \mapsto{ }^{\mathrm{t}} M J M
$$

For $G$-orbits $X_{J}, X_{J^{\prime}}, G$ acts on $X_{J} \times X_{J^{\prime}}$ by $\left(M, M^{\prime}\right)^{g}=\left(M^{g}, M^{\prime g}\right)$. The $G$-orbits on $X_{J} \times X_{J^{\prime}}$ are in one-to-one correspondence with the $G_{J}$-orbits on $X_{J^{\prime}}$, since the stabilizer of

$$
\left(\begin{array}{ll}
J & 0 \\
0 & 0
\end{array}\right) \in X_{J}
$$

in $G$ is $G_{J}$. By Lemma 2.2, $X_{J^{\prime}}$ is isomorphic to $O_{m^{\prime}}\left(q, J^{\prime}\right) \backslash \mathcal{M}_{m^{\prime}, n}^{*}(q)$, where $m^{\prime}$ is the matrix size of $J^{\prime}$. Let $O_{m^{\prime}}\left(q, J^{\prime}\right) \backslash \mathcal{M}_{m^{\prime}, n}^{*}(q) / G_{J}$ denote the $G_{J}$-orbits on $O_{m^{\prime}}\left(q, J^{\prime}\right) \backslash \mathcal{M}_{m^{\prime}, n}^{*}(q)$, i.e. the $\left(O_{m^{\prime}}\left(q, J^{\prime}\right) \times G_{J}\right)$-orbits on $\mathcal{M}_{m^{\prime}, n}^{*}(q)$. Then we have the following.

COROLlaRY 2.3. The G-orbits on $X_{J} \times X_{J^{\prime}}$ are in one-to-one correspondence with $O_{m^{\prime}}\left(q, J^{\prime}\right) \backslash \mathcal{M}_{m^{\prime}, n}^{*}(q) / G_{J}$.

Let us set

$$
H_{J}=\left\{g \in G \mid g=\left(\begin{array}{cc}
A & 0 \\
0 & I_{n-m}
\end{array}\right), A \in O_{m}(q, J)\right\},
$$




$$
\begin{gathered}
K_{m}(q)=\left\{g \in G \mid g=\left(\begin{array}{cc}
I_{m} & 0 \\
0 & C
\end{array}\right), C \in G L_{n-m}(q)\right\}, \\
U_{m}(q)=\left\{g \in G \mid g=\left(\begin{array}{cc}
I_{m} & 0 \\
B & I_{n-m}
\end{array}\right), B \in \mathcal{M}_{n-m, m}(q)\right\} .
\end{gathered}
$$

Then $G_{J}=U_{m}(q) \rtimes\left(H_{J} \times K_{m}(q)\right)$, the semi-direct product of $U_{m}(q)$ by $H_{J} \times K_{m}(q)$. We frequently use this decomposition later.

In the next section, we specialize $i=1$ so that $J$ is one of

$$
J_{1}^{+}=1, \quad J_{1}^{-}=\epsilon, \quad J_{2}^{+}=\left(\begin{array}{ll}
0 & 1 \\
1 & 0
\end{array}\right), \quad J_{2}^{-}=\left(\begin{array}{cc}
1 & 0 \\
0 & -\epsilon
\end{array}\right) .
$$

For $J=J_{1}^{ \pm}$, we have $O_{1}(q, J)=\{ \pm 1\}$. For $J=J_{2}^{+}$, we have

$$
O_{2}(q, J)=O_{2}^{+}(q)=\left\langle\left(\begin{array}{cc}
x & 0 \\
0 & x^{-1}
\end{array}\right),\left(\begin{array}{ll}
0 & 1 \\
1 & 0
\end{array}\right) \mid x \in G F(q)^{\times}\right\rangle,
$$

where $G F(q)^{\times}$is the set of non-zero elements of $G F(q)$. For $J=J_{2}^{-}$, we have

$$
O_{2}(q, J)=O_{2}^{-}(q)=\left\langle\left(\begin{array}{cc}
x & \epsilon y \\
y & x
\end{array}\right),\left(\begin{array}{cc}
1 & 0 \\
0 & -1
\end{array}\right)\left|\begin{array}{c}
x, y \in G F(q) \\
x^{2}-\epsilon y^{2}=1
\end{array}\right| .\right.
$$

Let $F$ denote the extension field of $G F(q)$ by $\sqrt{\epsilon}$, a square root of $\epsilon$. Since $F$ is a finite field of $q^{2}$ elements, we may use $G F\left(q^{2}\right)$ in place of $F$. Set

$$
P=\left(\begin{array}{cc}
1 & 1 \\
1 / \sqrt{\epsilon} & -1 / \sqrt{\epsilon}
\end{array}\right), \quad P^{-1}=\frac{1}{2}\left(\begin{array}{cc}
1 & \sqrt{\epsilon} \\
1 & -\sqrt{\epsilon}
\end{array}\right) .
$$

Then $P$ is an element of $G L_{2}\left(q^{2}\right)$, the set of non-singular matrices over $G F\left(q^{2}\right)$ of size 2. Observe that $J_{2}^{+}, J_{2}^{-}$are in the same orbit of $G L_{2}\left(q^{2}\right)$; in fact, it holds that ${ }^{\mathrm{t}} P J_{2}^{-} P=2 J_{2}^{+}$and 2 is a square in $G F\left(q^{2}\right)^{\times}$, since the mapping $\xi \mapsto \xi^{q+1}$ from $G F\left(q^{2}\right)^{\times}$to $G F(q)^{\times}$is surjective. Thus we have

$$
P^{-1} O_{2}\left(q, J_{2}^{-}\right) P \subset O_{2}\left(q^{2}, J_{2}^{+}\right) .
$$

Let $\sigma$ denote the generator of the Galois group of $G F\left(q^{2}\right)$ over $G F(q)$, i.e. $\sigma: \xi \mapsto \xi^{q}$. We use the following lemma frequently in the next section.

LEMMA 2.4 .

(i)

$$
\left\{P^{-1}\left(\begin{array}{l}
a \\
b
\end{array}\right) \mid a, b \in G F(q)\right\}=\left\{\left(\begin{array}{c}
\alpha \\
\alpha^{\sigma}
\end{array}\right) \mid \alpha \in G F\left(q^{2}\right)\right\} .
$$


(ii)

$$
\{(a, b) P \mid a, b \in G F(q)\}=\left\{\left(\alpha, \alpha^{\sigma}\right) \mid \alpha \in G F\left(q^{2}\right)\right\} .
$$

(iii)

$$
\left\{P^{-1}\left(\begin{array}{ll}
a & b \\
c & d
\end{array}\right) P \mid a, b, c, d \in G F(q)\right\}=\left\{\left(\begin{array}{cc}
\alpha & \beta \\
\beta^{\sigma} & \alpha^{\sigma}
\end{array}\right) \mid \alpha, \beta \in G F\left(q^{2}\right)\right\} .
$$

Proof.

(i) For $\alpha, \beta \in G F\left(q^{2}\right)$, set

$$
\left(\begin{array}{l}
a \\
b
\end{array}\right)=P\left(\begin{array}{l}
\alpha \\
\beta
\end{array}\right) .
$$

Then $a, b$ belong to $G F(q)$, i.e. $a^{\sigma}=a, b^{\sigma}=b$ if and only if

$$
P^{\sigma}\left(\begin{array}{l}
\alpha^{\sigma} \\
\beta^{\sigma}
\end{array}\right)=P\left(\begin{array}{l}
\alpha \\
\beta
\end{array}\right) .
$$

Since $\sqrt{\epsilon}^{\sigma}=-\sqrt{\epsilon}$, we have

$$
P^{-1} P^{\sigma}=\left(\begin{array}{ll}
0 & 1 \\
1 & 0
\end{array}\right)
$$

and the desired result.

(ii) The same proof as in (i) is valid.

(iii) For $\alpha, \beta, \gamma, \delta \in G F\left(q^{2}\right)$, set

$$
\left(\begin{array}{ll}
a & b \\
c & d
\end{array}\right)=P\left(\begin{array}{ll}
\alpha & \beta \\
\gamma & \delta
\end{array}\right) P^{-1}
$$

Then $a, b, c, d$ belong to $G F(q)$ if and only if

$$
P^{\sigma}\left(\begin{array}{ll}
\alpha^{\sigma} & \beta^{\sigma} \\
\gamma^{\sigma} & \delta^{\sigma}
\end{array}\right)\left(P^{-1}\right)^{\sigma}=P\left(\begin{array}{ll}
\alpha & \beta \\
\gamma & \delta
\end{array}\right) P^{-1} .
$$

Since

$$
P^{-1} P^{\sigma}=\left(\begin{array}{ll}
0 & 1 \\
1 & 0
\end{array}\right) \quad \text { and } \quad\left(P^{-1}\right)^{\sigma} P=\left(\begin{array}{ll}
0 & 1 \\
1 & 0
\end{array}\right)
$$

we obtain the desired result.

\section{Since}

$$
O_{2}\left(q^{2}, J_{2}^{+}\right)=\left\langle\left(\begin{array}{cc}
\xi & 0 \\
0 & \xi^{-1}
\end{array}\right),\left(\begin{array}{ll}
0 & 1 \\
1 & 0
\end{array}\right) \mid \xi \in G F\left(q^{2}\right)^{\times}\right\rangle,
$$

we have, by Lemma 2.4(iii),

$$
P^{-1} O_{2}\left(q, J_{2}^{-}\right) P=\left\langle\left(\begin{array}{cc}
\xi & 0 \\
0 & \xi^{-1}
\end{array}\right),\left(\begin{array}{ll}
0 & 1 \\
1 & 0
\end{array}\right) \mid \xi \in G F\left(q^{2}\right)^{\times}, \xi^{\sigma}=\xi^{-1}\right\rangle .
$$




\section{The $G$-orbits on $X_{1} \times X_{1}$}

We keep the same notation as in Sections 1 and 2. As we saw in Section 2, $G$ has four orbits on $X_{1}$. They are $X_{J}$ for $J \in\left\{J_{1}^{+}, J_{1}^{-}, J_{2}^{+}, J_{2}^{-}\right\}$. Recall that

$$
J_{1}^{+}=1, \quad J_{1}^{-}=\epsilon, \quad J_{2}^{+}=\left(\begin{array}{cc}
0 & 1 \\
1 & 0
\end{array}\right), \quad J_{2}^{-}=\left(\begin{array}{cc}
1 & 0 \\
0 & -\epsilon
\end{array}\right) .
$$

Recall that $X_{J}$ is the $G$-orbit containing $\left(\begin{array}{ll}J & 0 \\ 0 & 0\end{array}\right)$. Thus we have

$$
X_{1} \times X_{1}=\bigcup_{J, J^{\prime}} X_{J} \times X_{J^{\prime}} \quad \text { (disjoint union), }
$$

where $J, J^{\prime}$ run through $\left\{J_{1}^{ \pm}, J_{2}^{ \pm}\right\}$. In order to determine the $G$-orbits on $X_{1} \times X_{1}$, it suffices to determine the $G$-orbits on each $X_{J} \times X_{J^{\prime}}$. Note that the $G$-orbits on $X_{J} \times X_{J^{\prime}}$ and those on $X_{J^{\prime}} \times X_{J}$ are in one-to-one correspondence by the mapping $\left(M, M^{\prime}\right) \mapsto\left(M^{\prime}, M\right)$. Since $X_{J_{1}^{-}}=\epsilon X_{J_{1}^{+}}$, the $G$-orbits on $X_{J} \times X_{J_{1}^{+}}$and those on $X_{J} \times X_{J_{1}^{-}}$are in one-to-one correspondence by the mapping $\left(M, M^{\prime}\right) \mapsto\left(M, \epsilon M^{\prime}\right)$. Thus among the $16 X_{J} \times X_{J^{\prime}}$, we only need to treat $X_{J} \times X_{J^{\prime}}$ for $\left(J, J^{\prime}\right) \in$ $\left\{\left(J_{1}^{+}, J_{1}^{+}\right),\left(J_{2}^{ \pm}, J_{1}^{+}\right),\left(J_{2}^{+}, J_{2}^{ \pm}\right),\left(J_{2}^{-}, J_{2}^{-}\right)\right\}$.

By Corollary 2.3, we determine the $\left(O_{m^{\prime}}\left(q, J^{\prime}\right) \times G_{J}\right)$-orbits on $\mathcal{M}_{m^{\prime}, n}^{*}(q)$ instead of the $G$-orbits on $X_{J} \times X_{J^{\prime}}\left(m^{\prime}=1,2\right)$. For $M \in \mathcal{M}_{m^{\prime}, n}^{*}(q)$, write $M$ as $M=$ $\left(M_{0}, M_{1}\right)$ with $M_{0} \in \mathcal{M}_{m^{\prime}, m^{\prime}}(q), M_{1} \in \mathcal{M}_{m^{\prime}, n-m^{\prime}}(q)$. We call $M$ of type I, II, III, respectively (for $m^{\prime}=1$, only type I and II appear), as rank $M_{1}=m^{\prime}, m^{\prime}-1, m^{\prime}-2$. Let $\Omega_{\mathrm{I}}, \Omega_{\mathrm{II}}, \Omega_{\mathrm{III}}$ denote the set of matrices in $\mathcal{M}_{m^{\prime}, n}^{*}(q)$ of type I, II, III, respectively. Apparently, each of $\Omega_{\mathrm{I}}, \Omega_{\mathrm{II}}, \Omega_{\mathrm{III}}$ is fixed as a set by the action of $\left(O_{m^{\prime}}\left(q, J^{\prime}\right) \times G_{J}\right)$.

\subsection{The case $\left(J, J^{\prime}\right)=\left(J_{1}^{+}, J_{1}^{+}\right)$}

We apply Corollary 2.3 with $J=J^{\prime}=J_{1}^{+}, m^{\prime}=1, O_{m^{\prime}}\left(q, J^{\prime}\right)=\{ \pm 1\}$, $G_{J}=U_{1}(q) \rtimes\left(H_{J} \times K_{1}(q)\right)$, where

$$
H_{J}=\left\langle\left(\begin{array}{cc}
-1 & 0 \\
0 & I_{n-1}
\end{array}\right)\right\rangle .
$$

Apparently, we have the following theorem.

THEOREM 3.1. $O_{1}\left(q, J_{1}^{+}\right) \backslash \mathcal{M}_{1, n}^{*}(q) / G_{J_{1}^{+}}$consists of:

(i) $\quad\left\{(a, \mathbf{b}) \mid a \in G F(q), \mathbf{b} \in \mathcal{M}_{1, n-1}^{*}(q)\right\}$;

(ii) $\{(D, 0, \ldots, 0),(-D, 0, \ldots, 0)\}$ for $D \in G F(q)^{\times} / \sim$, where $G F(q)^{\times}=$ $G F(q)-\{0\}$, and $D^{\prime} \sim D$ is defined by $D^{\prime}= \pm D$.

There are $1+(q-1) / 2$ orbits. 
3.2. The case $\left(J, J^{\prime}\right)=\left(J_{2}^{+}, J_{1}^{+}\right)$

We apply Corollary 2.3 with

$$
\begin{gathered}
J=J_{2}^{+}, \quad J^{\prime}=J_{1}^{+}, \quad m^{\prime}=1, \quad O_{m^{\prime}}\left(q, J^{\prime}\right)=\{ \pm 1\}, \\
G_{J}=U_{2}(q) \rtimes\left(H_{J} \times K_{2}(q)\right), \quad H_{J}=\left\{\left(\begin{array}{cc}
A & 0 \\
0 & I_{n-2}
\end{array}\right) \mid A \in O_{2}^{+}(q)\right\} .
\end{gathered}
$$

THEOREM 3.2. $O_{1}\left(q, J_{1}^{+}\right) \backslash \mathcal{M}_{1, n}^{*}(q) / G_{J_{2}^{+}}$consists of:

(i) $\Omega_{\mathrm{I}}^{0}=\left\{(a, b, \mathbf{c}) \mid a, b \in G F(q), \mathbf{c} \in \mathcal{M}_{1, n-2}^{*}(q)\right\}$;

(ii) $\Omega_{\text {II }}^{+}(D)=\{(a, b, 0, \ldots, 0) \mid a, b \in G F(q), a b=D,(a, b) \neq(0,0)\}$ for $D \in G F(q)$.

There are $1+q$ orbits.

Proof. Apparently, $\Omega_{\mathrm{I}}^{0}$ and $\Omega_{\mathrm{II}}^{+}(D)$ are fixed as a set by the action of $O_{1}\left(q, J_{1}^{+}\right)=$ $\{ \pm 1\}$ and that of $G_{J}=U_{2}(q) \rtimes\left(H_{J} \times K_{2}(q)\right)$.

Every element of $\Omega_{\mathrm{I}}^{0}$ is sent to $(a, b, 1,0, \ldots, 0)$ for some $a, b \in G F(q)$ by the action of $K_{2}(q)$. Any element $(a, b, 1,0, \ldots, 0)$ is sent to $(0,0,1,0, \ldots, 0)$ by $U_{2}(q)$. Thus $\Omega_{\mathrm{I}}^{0}$ is an orbit.

Every element of $\Omega_{\mathrm{II}}^{+}(D)$ is sent to $(1, D, 0, \ldots, 0)$ by the action of $H_{J}$, since $O_{2}^{+}(q)$ is generated by

$$
\left(\begin{array}{cc}
x & 0 \\
0 & x^{-1}
\end{array}\right)\left(x \in G F(q)^{\times}\right) \text {and } \quad\left(\begin{array}{ll}
0 & 1 \\
1 & 0
\end{array}\right) .
$$

So $\Omega_{\mathrm{II}}^{+}(D)$ is an orbit.

\subsection{The case $\left(J, J^{\prime}\right)=\left(J_{2}^{-}, J_{1}^{+}\right)$}

We apply Corollary 2.3 with

$$
\begin{gathered}
J=J_{2}^{-}, \quad J^{\prime}=J_{1}^{+}, \quad m^{\prime}=1, \quad O_{m^{\prime}}\left(q, J^{\prime}\right)=\{ \pm 1\}, \\
G_{J}=U_{2}(q) \rtimes\left(H_{J} \times K_{2}(q)\right), \quad H_{J}=\left\{\left(\begin{array}{cc}
A & 0 \\
0 & I_{n-2}
\end{array}\right) \mid A \in O_{2}^{-}(q)\right\} .
\end{gathered}
$$

Recall that

$$
P=\left(\begin{array}{cc}
1 & 1 \\
1 / \sqrt{\epsilon} & -1 / \sqrt{\epsilon}
\end{array}\right), \quad P^{-1}=\frac{1}{2}\left(\begin{array}{cc}
1 & \sqrt{\epsilon} \\
1 & -\sqrt{\epsilon}
\end{array}\right), \quad\langle\sigma\rangle=\operatorname{Gal}\left(G F\left(q^{2}\right) / G F(q)\right),
$$


and the Galois group of $G F\left(q^{2}\right)$ over $G F(q)$. Set

$$
\widetilde{P}=\left(\begin{array}{cc}
P & 0 \\
0 & I_{n-2}
\end{array}\right) \in G L_{n}\left(q^{2}\right) .
$$

THEOREM 3.3. $O_{1}\left(q, J_{1}^{+}\right) \backslash \mathcal{M}_{1, n}^{*}(q) / G_{J_{2}^{-}}$consists of:

(i) $\quad \Omega_{\mathrm{I}}^{0}=\left\{(a, b, \mathbf{c}) \mid a, b \in G F(q), \mathbf{c} \in \mathcal{M}_{1, n-2}^{*}(q)\right\}$;

(ii) $\Omega_{\mathrm{II}}^{-}(D)=\Lambda_{\mathrm{II}}^{-}(D) \widetilde{P}^{-1}$ for $D \in G F(q)^{\times}$, where

$$
\Lambda_{\mathrm{II}}^{-}(D)=\left\{\left(\alpha, \alpha^{\sigma}, 0, \ldots, 0\right) \mid \alpha \in G F\left(q^{2}\right)^{\times}, \alpha \alpha^{\sigma}=D\right\} .
$$

There are $1+(q-1)$ orbits.

Proof. The argument in the proof of Theorem 3.2 is valid for $\Omega_{\mathrm{I}}^{0}$. So $\Omega_{\mathrm{I}}^{0}$ is an orbit.

We have $\mathcal{M}_{1, n}^{*}(q)-\Omega_{\mathrm{I}}^{0}=\{(a, b, 0, \ldots, 0) \mid a, b \in G F(q),(a, b) \neq(0,0)\}$, and $K_{2}(q), U_{2}(q)$ act on it trivially. The action of $H_{J}$ on $\mathcal{M}_{1, n}^{*}(q)-\Omega_{\mathrm{I}}^{0}$ is isomorphic to that of $O_{2}^{-}(q)$ on $\mathcal{M}_{1,2}^{*}(q)$. The action of $O_{2}^{-}(q)$ on $\mathcal{M}_{1,2}^{*}(q)$ is isomorphic to that of $P^{-1} O_{2}^{-}(q) P$ on $\mathcal{M}_{1,2}^{*}(q) P$ by the correspondence $(M, A) \mapsto\left(M P, P^{-1} A P\right)$ from $\mathcal{M}_{1,2}^{*} \times O_{2}^{-}(q)$ to $\mathcal{M}_{1,2}^{*}(q) P \times P^{-1} O_{2}^{-}(q) P$. By Lemma 2.4(ii), we have $\mathcal{M}_{1,2}^{*} P=\left\{\left(\alpha, \alpha^{\sigma}\right) \mid \alpha \in G F\left(q^{2}\right)^{\times}\right\}$. As we saw in Section 2,

$$
P^{-1} O_{2}^{-}(q) P=\left\langle\left(\begin{array}{cc}
\xi & 0 \\
0 & \xi^{-1}
\end{array}\right),\left(\begin{array}{ll}
0 & 1 \\
1 & 0
\end{array}\right) \mid \xi \in G F\left(q^{2}\right)^{\times}, \xi^{\sigma}=\xi^{-1}\right\rangle .
$$

So the orbits of $P^{-1} O_{2}^{-}(q) P$ on $\mathcal{M}_{1,2}^{*} P$ are

$$
\Lambda(D)=\left\{\left(\alpha, \alpha^{\sigma}\right) \mid \alpha \in G F\left(q^{2}\right), \alpha \alpha^{\sigma}=D\right\} \quad \text { for } \quad D \in G F(q)^{\times} .
$$

Here we have used the fact that the mapping $\xi \mapsto \xi \xi^{\sigma}$ is a surjection from $G F\left(q^{2}\right)^{\times}$ to $G F(q)^{\times}$. Thus $\Lambda(D) P^{-1}\left(D \in G F(q)^{\times}\right)$are the orbits of $O_{2}^{-}(q)$ on $\mathcal{M}_{1,2}^{*}(q)$ and the theorem follows.

\subsection{The case $\left(J, J^{\prime}\right)=\left(J_{2}^{+}, J_{2}^{+}\right)$}

We apply Corollary 2.3 with

$$
\begin{gathered}
J=J^{\prime}=J_{2}^{+}, \quad m^{\prime}=2, \quad O_{m^{\prime}}\left(q, J^{\prime}\right)=O_{2}^{+}(q), \\
G_{J}=U_{2}(q) \rtimes\left(H_{J} \times K_{2}(q)\right), \quad H_{J}=\left\{\left(\begin{array}{cc}
A & 0 \\
0 & I_{n-2}
\end{array}\right) \mid A \in O_{2}^{+}(q)\right\} .
\end{gathered}
$$

Let $\chi$ denote the quadratic residue character of $G F(q)$, i.e. $\chi(x)=0,1$ or -1 , respectively, as $x$ is 0 , a square of $G F(q)^{\times}$or a non-square of $G F(q)^{\times}$. 
THEOREM 3.4. $O_{2}\left(q, J_{2}^{+}\right) \backslash \mathcal{M}_{2, n}^{*}(q) / G_{J_{2}^{+}}$consists of:

(i) $\Omega_{\mathrm{I}}=\left\{\left(M_{0}, M_{1}\right) \mid M_{0} \in \mathcal{M}_{2,2}(q), M_{1} \in \mathcal{M}_{2, n-2}^{*}(q)\right\}$;

(ii) $\Omega_{\mathrm{II}}^{++}\left(D_{1}, D_{2}\right)=\Lambda_{\mathrm{II}}^{++}\left(D_{1}, D_{2}\right) K_{2}(q)$ for $\left(D_{1}, D_{2}\right) \in G F(q) \times G F(q) / \sim$, where

$$
\begin{aligned}
& \Lambda_{\mathrm{II}}^{++}\left(D_{1}, D_{2}\right)=\left\{(\mathbf{a}, \mathbf{b}, \mathbf{c}, 0, \ldots, 0) \mid(\mathbf{a}, \mathbf{b}, \mathbf{c}) \in \mathcal{M}_{2,3}^{*}(q), \mathbf{c}=\left(\begin{array}{l}
c_{1} \\
c_{2}
\end{array}\right) \neq\left(\begin{array}{l}
0 \\
0
\end{array}\right),\right. \\
&\left.\left(\operatorname{det}(\mathbf{a}, \mathbf{c}) \operatorname{det}(\mathbf{b}, \mathbf{c}), c_{1} c_{2}\right) \sim\left(D_{1}, D_{2}\right)\right\}, \\
& \text { and }\left(D_{1}, D_{2}\right) \sim\left(D_{1}^{\prime}, D_{2}^{\prime}\right) \Leftrightarrow D_{1}^{\prime}=x^{2} D_{1}, D_{2}^{\prime}=x^{2} D_{2} \text { for some } x \in G F(q)^{\times} ;
\end{aligned}
$$

(iii)

$$
\begin{aligned}
\Omega_{\mathrm{III}}^{++}\left(\left\{D_{1}, D_{2}\right\},\left\{\kappa_{1}, \kappa_{2}\right\}\right)= & \left\{\left(\begin{array}{ll}
a & c \\
b & d
\end{array}\right) \in \mathcal{M}_{2, n}^{*}(q) \mid\{a d, b c\}=\left\{D_{1}, D_{2}\right\},\right. \\
& \left.\{\chi(a b), \chi(c d)\}=\left\{\kappa_{1}, \kappa_{2}\right\} \text { as multisets }\right\}
\end{aligned}
$$

for $D_{1}, D_{2} \in G F(q)\left(D_{1} \neq D_{2}\right), \kappa_{1}, \kappa_{2} \in\{0,1,-1\}$ such that $\kappa_{1} \kappa_{2}=$ $\chi\left(D_{1} D_{2}\right)$.

There are $1+(2 q+3)+\frac{1}{4}(q-1)(3 q+5)$ orbits.

Proof. It is easily checked that the action of $O_{2}^{+}(q)$ fixes each of $\Omega_{\mathrm{I}}, \Omega_{\mathrm{II}}^{++}\left(D_{1}, D_{2}\right)$, $\Omega_{\text {III }}^{++}\left(\left\{D_{1}, D_{2}\right\},\left\{\kappa_{1}, \kappa_{2}\right\}\right)$ as a set. Also $U_{2}(q), K_{2}(q), H_{J}$ fix each of $\Omega_{\mathrm{I}}$, $\Omega_{\mathrm{II}}^{++}\left(D_{1}, D_{2}\right), \Omega_{\mathrm{III}}^{++}\left(\left\{D_{1}, D_{2}\right\},\left\{\kappa_{1}, \kappa_{2}\right\}\right)$ as a set, and so $G_{J}$ fixes each of them as a set.

Every element of $\Omega_{\mathrm{I}}$ is sent to $\left(M_{0}, I, \mathbf{0}\right)$ for some $M_{0} \in \mathcal{M}_{2,2}(q)$ by $K_{2}(q)$, where

$$
I=\left(\begin{array}{ll}
1 & 0 \\
0 & 1
\end{array}\right) .
$$

Any element $\left(M_{0}, I, \mathbf{0}\right)$ is sent to $(0, I, \mathbf{0})$ by $U_{2}(q)$. So, $\Omega_{\mathrm{I}}$ is an orbit.

Every element of $\Omega_{\mathrm{II}}^{++}\left(D_{1}, D_{2}\right)$ is, by the action of $K_{2}(q)$, sent to $(\mathbf{a}, \mathbf{b}, \mathbf{c}, \mathbf{0})$ such that

$$
\mathbf{c}=\left(\begin{array}{l}
c_{1} \\
c_{2}
\end{array}\right) \neq\left(\begin{array}{l}
0 \\
0
\end{array}\right)
$$

with $c_{1} c_{2}=D_{2}$. By the action of $O_{2}^{+}(q)$, we may assume that $c_{1}=D_{2}, c_{2}=1$. Any such element $(\mathbf{a}, \mathbf{b}, \mathbf{c}, \mathbf{0})$ is sent to $\left(\begin{array}{ccc}a_{1} & b_{1} & D_{2} \\ 0 & 0 & 0\end{array}\right)$ for some $a_{1}, b_{1} \in G F(q)$ by the action of $U_{2}(q)$. Since it belongs to $\Omega_{\mathrm{II}}^{++}\left(D_{1}, D_{2}\right)$, we have $a_{1} b_{1}=D_{1}$. The action of $H_{J}$ sends $\left(\begin{array}{ccc}a_{1} & b_{1} & D_{2} \\ 0 & 0 & 1\end{array}\right)$ to $\left(\begin{array}{cccc}1 & D_{1} & D_{2} \\ 0 & 0 & 1 & 0\end{array}\right)$. Thus $\Omega_{\mathrm{II}}^{++}\left(D_{1}, D_{2}\right)$ is an orbit. 
For

$$
\left(\begin{array}{ll}
a & c \\
b & d
\end{array}\right) \in \Omega_{\mathrm{III}}^{++}\left(\left\{D_{1}, D_{2}\right\},\left\{\kappa_{1}, \kappa_{2}\right\}\right),
$$

we may assume that $a d=D_{1}, b c=D_{2}, \chi(a b)=\kappa_{1}, \chi(c d)=\kappa_{2}$ by applying

$$
\left(\begin{array}{ll}
0 & 1 \\
1 & 0
\end{array}\right) \in O_{2}^{+}(q), \quad\left(\begin{array}{cc|c}
0 & 1 & \mathbf{0} \\
1 & 0 & \mathbf{0} \\
\hline \mathbf{0} & \mathbf{0} & I_{n-2}
\end{array}\right) \in H_{J}
$$

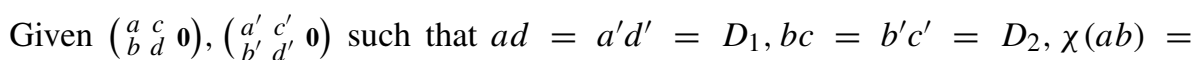
$\chi\left(a^{\prime} b^{\prime}\right)=\kappa_{1}, \chi(c d)=\chi\left(c^{\prime} d^{\prime}\right)=\kappa_{2}$, we claim that there exist $x, y \in G F(q)^{\times}$ such that

$$
\left(\begin{array}{cc}
x & 0 \\
0 & x^{-1}
\end{array}\right)\left(\begin{array}{ll}
a & c \\
b & d
\end{array}\right)\left(\begin{array}{cc}
y & 0 \\
0 & y^{-1}
\end{array}\right)=\left(\begin{array}{ll}
a^{\prime} & c^{\prime} \\
b^{\prime} & d^{\prime}
\end{array}\right),
$$

i.e. $a^{\prime}=x y a, b^{\prime}=x^{-1} y b, c^{\prime}=x y^{-1} c, d^{\prime}=x^{-1} y^{-1} d$. Suppose that $\kappa_{1} \neq 0$. Then $a, b, a^{\prime}, b^{\prime}$ are non-zero by $\chi(a b)=\chi\left(a^{\prime} b^{\prime}\right)=\kappa_{1}$. Let $\sqrt{a}, \sqrt{b}, \sqrt{a^{\prime}}, \sqrt{b^{\prime}} \in$ $G F\left(q^{2}\right)^{\times}$be square roots of $a, b, a^{\prime}, b^{\prime}$ and set $x=\sqrt{a^{\prime}} \sqrt{b} / \sqrt{a} \sqrt{b^{\prime}}, y=$ $\sqrt{a^{\prime}} \sqrt{b^{\prime}} / \sqrt{a} \sqrt{b}$. Then $x^{2}=a^{\prime} b / a b^{\prime}, y^{2}=a^{\prime} b^{\prime} / a b$ are squares in $G F(q)^{\times}$by $\chi(a b)=\chi\left(a^{\prime} b^{\prime}\right)$. So $x, y$ are contained in $G F(q)^{\times}$. It holds that $x y a=a^{\prime}, x^{-1} y b=$ $b^{\prime}, x y^{-1} c=b c / b^{\prime}=c^{\prime}, x^{-1} y^{-1} d=a d / a^{\prime}=d^{\prime}$, since $b c=b^{\prime} c^{\prime}=D_{2}, a d=$ $a^{\prime} d^{\prime}=D_{1}$. So $x, y$ are desired. Suppose that $\kappa_{2} \neq 0$. Then $c, d, c^{\prime}, d^{\prime}$ are nonzero and $x=\sqrt{c^{\prime}} \sqrt{d} / \sqrt{c} \sqrt{d^{\prime}}, y=\sqrt{c} \sqrt{d} / \sqrt{c^{\prime}} \sqrt{d^{\prime}}$ are desired. Suppose that $\kappa_{1}=\kappa_{2}=0$. If $D_{1} \neq 0$, then $a d=a^{\prime} d^{\prime}=D_{1} \neq 0, b=c=0, b^{\prime}=c^{\prime}=0$, and so $x=a^{\prime} / a=d / d^{\prime}, y=1$ meet the requirement. If $D_{2} \neq 0$, then $b c=b^{\prime} c^{\prime}=D_{2} \neq 0$, $a=d=0, a^{\prime}=d^{\prime}=0$, and so $x=b / b^{\prime}=c^{\prime} / c, y=1$ meet the requirement. The case $D_{1}=D_{2}=0$ does not occur, since $D_{1} \neq D_{2}$ due to the non-singularity of the matrix $\left(\begin{array}{ll}a & c \\ b & d\end{array}\right)$. Thus the claim is proved and $\Omega_{\mathrm{III}}^{++}\left(\left\{D_{1}, D_{2}\right\},\left\{\kappa_{1}, \kappa_{2}\right\}\right)$ is an orbit.

Apparently, each element of $\mathcal{M}_{2, n}^{*}(q)$ belongs to exactly one of $\Omega_{\mathrm{I}}$, $\Omega_{\mathrm{II}}^{++}\left(D_{1}, D_{2}\right), \Omega_{\mathrm{III}}^{++}\left(\left\{D_{1}, D_{2}\right\},\left\{\kappa_{1}, \kappa_{2}\right\}\right)$. Thus they are the orbits of $O_{2}^{+}(q) \times G_{J_{2}^{+}}$ on $\mathcal{M}_{2, n}^{*}(q)$.

For the orbits $\Omega_{\mathrm{II}}^{++}\left(D_{1}, D_{2}\right)$, the parameter $\left(D_{1}, D_{2}\right)$ ranges over $G F(q) \times$ $G F(q) / \sim$, where $\left(D_{1}, D_{2}\right) \sim\left(D_{1}^{\prime}, D_{2}^{\prime}\right) \Leftrightarrow D_{1}^{\prime}=x^{2} D_{1}, D_{2}^{\prime}=x^{2} D_{2}$ for some $x \in G F(q)^{\times}$. For $\left(D_{1}, D_{2}\right) \neq(0,0)$, the equivalence class to which $\left(D_{1}, D_{2}\right)$ belongs contains $(q-1) / 2$ elements, while $(0,0)$ is itself an equivalence class. Since $1+2\left(q^{2}-1\right) /(q-1)=2 q+3$, there are $2 q+3$ equivalence classes, i.e. $2 q+3$ orbits $\Omega_{\mathrm{II}}^{++}\left(D_{1}, D_{2}\right)$. 
For the orbits $\Omega_{\mathrm{III}}^{++}\left(\left\{D_{1}, D_{2}\right\},\left\{\kappa_{1}, \kappa_{2}\right\}\right)$, the parameter $\left(\left\{D_{1}, D_{2}\right\},\left\{\kappa_{1}, \kappa_{2}\right\}\right)$ ranges over $D_{1}, D_{2} \in G F(q)\left(D_{1} \neq D_{2}\right), \kappa_{1}, \kappa_{2} \in\{0,1,-1\}\left(\kappa_{1} \kappa_{2}=\chi\left(D_{1} D_{2}\right)\right)$. The number of $\left\{D_{1}, D_{2}\right\}$ such that $D_{1}, D_{2}$ are distinct squares in $G F(q)^{\times}$ is $\frac{1}{2}((q-1) / 2)((q-1) / 2-1)$. For each of such $\left\{D_{1}, D_{2}\right\}$, the choice of $\left\{\kappa_{1}, \kappa_{2}\right\}$ such that $\kappa_{1} \kappa_{2}=\chi\left(D_{1} D_{2}\right)$ is either $\{1,1\}$ or $\{-1,-1\}$. Thus there are $(q-1) / 2((q-1) / 2-1)$ parameters of this type. Similarly there are $(q-1) / 2((q-1) / 2-1)$ parameters $\left(\left\{D_{1}, D_{2}\right\},\left\{\kappa_{1}, \kappa_{2}\right\}\right)$ such that $D_{1}, D_{2}$ are distinct non-squares in $G F(q)^{\times}$. The number of $\left\{D_{1}, D_{2}\right\}$ such that one of $D_{1}, D_{2}$ is a square in $G F(q)^{\times}$and the other is a non-square in $G F(q)^{\times}$is $(q-1) / 2((q-1) / 2)$. For each of such $\left\{D_{1}, D_{2}\right\}$, the choice of $\left\{\kappa_{1}, \kappa_{2}\right\}$ such that $\kappa_{1} \kappa_{2}=\chi\left(D_{1} D_{2}\right)$ is only $\{1,-1\}$. Thus there are $(q-1) / 2((q-1) / 2)$ parameters of this type. The number of $\left\{D_{1}, D_{2}\right\}$ such that one of $D_{1}, D_{2}$ is zero and the other is non-zero is $q-1$. For each of such $\left\{D_{1}, D_{2}\right\}$, the choice of $\left\{\kappa_{1}, \kappa_{2}\right\}$ such that $\kappa_{1} \kappa_{2}=\chi\left(D_{1} D_{2}\right)$ is $\{0,0\},\{0,1\}$ or $\{0,-1\}$. Thus there are $3(q-1)$ parameters of this type. Therefore the number of orbits $\Omega_{\text {III }}^{++}\left(\left\{D_{1}, D_{2}\right\},\left\{\kappa_{1}, \kappa_{2}\right\}\right)$ is

$$
\begin{aligned}
& \frac{q-1}{2}\left(\frac{q-1}{2}-1\right)+\frac{q-1}{2}\left(\frac{q-1}{2}-1\right)+\frac{q-1}{2} \frac{q-1}{2}+3(q-1) \\
& =\frac{1}{4}(q-1)(3 q+5) .
\end{aligned}
$$

3.5. The case $\left(J, J^{\prime}\right)=\left(J_{2}^{+}, J_{2}^{-}\right)$

We apply Corollary 2.3 with

$$
\begin{gathered}
J=J_{2}^{+}, \quad J^{\prime}=J_{2}^{-}, \quad m^{\prime}=2, \quad O_{m^{\prime}}\left(q, J^{\prime}\right)=O_{2}^{-}(q), \\
G_{J}=U_{2}(q) \rtimes\left(H_{J} \times K_{2}(q)\right), \quad H_{J}=\left\{\left(\begin{array}{cc}
A & 0 \\
0 & I_{n-2}
\end{array}\right) \mid A \in O_{2}^{+}(q)\right\} .
\end{gathered}
$$

Let $\chi$ denote the quadratic residue character of $G F(q)$.

Recall that

$$
P=\left(\begin{array}{cc}
1 & 1 \\
1 / \sqrt{\epsilon} & -1 / \sqrt{\epsilon}
\end{array}\right)
$$

and $\langle\sigma\rangle=\operatorname{Gal}\left(G F\left(q^{2}\right) / G F(q)\right)$, the Galois group of $G F\left(q^{2}\right)$ over $G F(q)$.

THEOREM 3.5. $O_{2}\left(q, J_{2}^{-}\right) \backslash \mathcal{M}_{2, n}^{*}(q) / G_{J_{2}^{+}}$consists of:

(i) $\Omega_{\mathrm{I}}=\left\{\left(M_{0}, M_{1}\right) \mid M_{0} \in \mathcal{M}_{2,2}(q), M_{1} \in \mathcal{M}_{2, n-2}^{*}(q)\right\}$; 
(ii) $\Omega_{\mathrm{II}}^{+-}(D, \kappa)=P \Lambda_{\mathrm{II}}^{+-}(D, \kappa) K_{2}(q)$; for $(D, \kappa) \in G F(q) \times\{ \pm 1\}$, where

$$
\begin{aligned}
\Lambda_{\mathrm{II}}^{+-}(D, \kappa)=\{ & \left(\begin{array}{ccc}
\alpha & \beta & \gamma \\
\alpha^{\sigma} & \beta^{\sigma} & \gamma^{\sigma}
\end{array}\right) \in \mathcal{M}_{2, n}^{*}\left(q^{2}\right) \mid \chi\left(\gamma \gamma^{\sigma}\right)=\kappa, \\
& \left.\frac{\left(\alpha \gamma^{\sigma}-\alpha^{\sigma} \gamma\right)\left(\beta \gamma^{\sigma}-\beta^{\sigma} \gamma\right)}{\gamma \gamma^{\sigma}}=D\right\} ;
\end{aligned}
$$

(iii) $\Omega_{\mathrm{III}}^{+-}\left(\left\{D, D^{\sigma}\right\},\left\{\kappa_{1}, \kappa_{2}\right\}\right)=P \Lambda_{\mathrm{III}}^{+-}\left(\left\{D, D^{\sigma}\right\},\left\{\kappa_{1}, \kappa_{2}\right\}\right)$ for $D \in G F\left(q^{2}\right)^{\times}-$ $G F(q)^{\times}, \kappa_{1}, \kappa_{2} \in\{ \pm 1\}$ such that $\chi\left(D D^{\sigma}\right)=\kappa_{1} \kappa_{2}$, where

$$
\begin{aligned}
\Lambda_{\mathrm{III}}^{+-}\left(\left\{D, D^{\sigma}\right\},\left\{\kappa_{1}, \kappa_{2}\right\}\right)=\{ & \left(\begin{array}{cc}
\alpha & \beta \\
\alpha^{\sigma} & \beta^{\sigma}
\end{array}\right) \in \mathcal{M}_{2, n}^{*}\left(q^{2}\right) \mid\left\{\alpha \beta^{\sigma}, \alpha^{\sigma} \beta\right\} \\
& =\left\{D, D^{\sigma}\right\},\left\{\chi\left(\alpha \alpha^{\sigma}\right), \chi\left(\beta \beta^{\sigma}\right)\right\} \\
& \left.=\left\{\kappa_{1}, \kappa_{2}\right\} \text { as multisets }\right\}
\end{aligned}
$$

There are $1+2 q+\frac{1}{4}(q-1)(3 q-1)$ orbits.

Proof. For $M \in \mathcal{M}_{2, n}^{*}(q)$, write $M$ as $M=\left(M_{0}, M_{1}\right)$ with $M_{0} \in \mathcal{M}_{2,2}(q), M_{1} \in$ $\mathcal{M}_{2, n-2}(q)$. Recall that we call $M$ of type I, II, III, respectively, as rank $M_{1}=2,1,0$. Let $\Omega_{\mathrm{I}}, \Omega_{\mathrm{II}}, \Omega_{\mathrm{III}}$ denote the set of matrices in $\mathcal{M}_{2, n}^{*}(q)$ of type I, II, III, respectively. Apparently, each of $\Omega_{\mathrm{I}}, \Omega_{\mathrm{II}}, \Omega_{\mathrm{III}}$ is fixed as a set by $O_{2}^{-}(q), G_{J}$.

The argument in the proof of Theorem 3.4 is valid for $\Omega_{\mathrm{I}}$. So $\Omega_{\mathrm{I}}$ is an orbit of $G_{J}$, and hence of $O_{2}^{-}(q) \times G_{J}$.

Each of $\Omega_{\mathrm{II}}^{+-}(D, \kappa), \Omega_{\mathrm{III}}^{+-}\left(\left\{D, D^{\sigma}\right\},\left\{\kappa_{1}, \kappa_{2}\right\}\right)$ is fixed as a set by each of $U_{2}(q)$, $K_{2}(q), H_{J}$ and hence by $G_{J}$. Also, each of them is fixed as a set by $O_{2}^{-}(q)$; to see this fact, note that the action of $O_{2}^{-}(q)$ on $\mathcal{M}_{2, n}^{*}(q)$ is isomorphic to that of $P^{-1} O_{2}^{-}(q) P$ on $P^{-1} \mathcal{M}_{2, n}^{*}(q)$ by the correspondence $(A, M) \mapsto\left(P^{-1} A P, P^{-1} M\right)$ from $O_{2}^{-}(q) \times \mathcal{M}_{2, n}^{*}(q)$ to $P^{-1} O_{2}^{-}(q) P \times P^{-1} \mathcal{M}_{2, n}^{*}(q)$, and check each of $\Lambda_{\mathrm{II}}^{+-}(D, \kappa), \Lambda_{\mathrm{III}}^{+-}\left(\left\{D, D^{\sigma}\right\},\left\{\kappa_{1}, \kappa_{2}\right\}\right)$ is fixed as a set by $P^{-1} O_{2}^{-}(q) P$, using

$$
P^{-1} O_{2}^{-}(q) P=\left\langle\left(\begin{array}{cc}
\xi & 0 \\
0 & \xi^{-1}
\end{array}\right),\left(\begin{array}{ll}
0 & 1 \\
1 & 0
\end{array}\right) \mid \xi \in G F\left(q^{2}\right)^{\times}, \xi^{\sigma}=\xi^{-1}\right\rangle .
$$

To show that $\Omega_{\mathrm{II}}^{+-}(D, \kappa)$ is an orbit of $O_{2}^{-}(q) \times G_{J}$, it suffices to show that $P^{-1} O_{2}^{-}(q) P \times U_{2}(q) H_{J}$ is transitive on $\Lambda_{\mathrm{II}}^{+-}(D, \kappa) K_{2}(q)$. Note that the mapping $\xi \mapsto \xi \xi^{\sigma}$ from $G F\left(q^{2}\right)^{\times}$to $G F(q)^{\times}$is surjective and has the kernel $\left\{\xi \in G F\left(q^{2}\right)^{\times} \mid\right.$ $\left.\xi^{\sigma}=\xi^{-1}\right\}$. Fix $\gamma_{0} \in G F\left(q^{2}\right)^{\times}$such that $\chi\left(\gamma_{0} \gamma_{0}^{\sigma}\right)=\kappa$. Then, since any $\gamma \in G F\left(q^{2}\right)^{\times}$such that $\chi\left(\gamma \gamma^{\sigma}\right)=\kappa$ can be written as $\gamma=\xi t \gamma_{0}$ for some 
$t \in G F(q)^{\times}, \xi \in G F\left(q^{2}\right)^{\times}$with $\xi^{\sigma}=\xi^{-1}$, every element of $\Lambda_{\mathrm{II}}^{+-}(D, \kappa)$ is sent to

$$
\left(\begin{array}{cccc}
\alpha & \beta & \gamma_{0} & \\
\alpha^{\sigma} & \beta^{\sigma} & \gamma_{0}^{\sigma} & \mathbf{0}
\end{array}\right) \in \Lambda_{\mathrm{II}}^{+-}(D, \kappa)
$$

for some $\alpha, \beta \in G F\left(q^{2}\right)$ by the action of $P^{-1} O_{2}^{-}(q) P$ and $K_{2}(q)$. Then we have

$$
\frac{\alpha}{\gamma_{0}}-\left(\frac{\alpha}{\gamma_{0}}\right)^{\sigma}=a \sqrt{\epsilon}, \quad \frac{\beta}{\gamma_{0}}-\left(\frac{\beta}{\gamma_{0}}\right)^{\sigma}=b \sqrt{\epsilon}
$$

for some $a, b \in G F(q)$, since any element $\xi \in G F\left(q^{2}\right)$ such that $\xi^{\sigma}=-\xi$ can be written as $\xi=c \sqrt{\epsilon}$ for some $c \in G F(q)$. By the action of $H_{J}$, we may assume that $a=1, b=D / \epsilon \gamma_{0} \gamma_{0}^{\sigma}$, since

$$
\left(\frac{\alpha}{\gamma_{0}}-\frac{\alpha^{\sigma}}{\gamma_{0}^{\sigma}}\right)\left(\frac{\beta}{\gamma_{0}}-\frac{\beta^{\sigma}}{\gamma_{0}^{\sigma}}\right)=\frac{D}{\gamma_{0} \gamma_{0}^{\sigma}} \text { and } a=b=0
$$

cannot occur due to the condition that $\left(\begin{array}{ccc}\alpha & \beta & \gamma_{0} \\ \alpha^{\sigma} & \beta^{\sigma} & \gamma_{0}^{\sigma}\end{array}\right)$ is of rank 2. Since the mapping $\xi \mapsto\left(\xi-\xi^{\sigma}\right) / \sqrt{\epsilon}$ from $G F\left(q^{2}\right)$ to $G F(q)$, i.e. the mapping $x+y \sqrt{\epsilon} \mapsto 2 y$ $(x, y \in G F(q))$, is surjective and has the kernel $G F(q)$ as an additive group, the equation $\xi-\xi^{\sigma}=a \sqrt{\epsilon}$ has a unique solution $\xi_{0} \bmod G F(q)$, i.e. $\alpha=\alpha_{0}+s \gamma_{0}$ $\left(\xi=\alpha / \gamma_{0}, \xi_{0}=\alpha_{0} / \gamma_{0}, s \in G F(q)\right)$. Also, the equation $\xi-\xi^{\sigma}=b \sqrt{\epsilon}$ has a unique solution $\xi_{1} \bmod G F(q)$, i.e. $\beta=\beta+t \gamma_{0}\left(\xi=\beta / \gamma_{0}, \xi_{1}=\beta_{0} / \gamma_{0}, t \in G F(q)\right)$. By the action of $U_{2}(q),\left(\begin{array}{cccc}\alpha & \beta & \gamma_{0} & 0 \\ \alpha^{\sigma} & \beta^{\sigma} & \gamma_{0}^{\sigma} & 0\end{array}\right)$ is sent to $\left(\begin{array}{cccc}\alpha_{0} & \beta_{0} & \gamma_{0} \\ \alpha_{0}^{\sigma} & \beta_{0}^{\sigma} & \gamma_{0}^{\sigma} & \mathbf{0}\end{array}\right)$. Thus $P^{-} O_{2}^{-}(q) P \times U_{2}(q) H_{J}$ is transitive on $\Lambda_{\mathrm{II}}^{+-}(D, \kappa) K_{2}(q)$.

To show that $\Omega_{\mathrm{III}}^{+-}\left(\left\{D, D^{\sigma}\right\},\left\{\kappa_{1}, \kappa_{2}\right\}\right)$ is an orbit of $O_{2}^{-}(q) \times G_{J}$, it suffices to show that $P^{-1} O_{2}^{-}(q) P \times H_{J}$ is transitive on $\Lambda_{\mathrm{III}}^{+-}\left(\left\{D, D^{\sigma}\right\},\left\{\kappa_{1}, \kappa_{2}\right\}\right)$. For

$$
\left(\begin{array}{ccc}
\alpha & \beta & \\
\alpha^{\sigma} & \beta^{\sigma} & \mathbf{0}
\end{array}\right) \in \Lambda_{\mathrm{III}}^{+-}\left(\left\{D, D^{\sigma}\right\},\left\{\kappa_{1}, \kappa_{2}\right\}\right),
$$

we may assume that $\alpha \beta^{\sigma}=D, \alpha^{\sigma} \beta=D^{\sigma}, \chi\left(\alpha \alpha^{\sigma}\right)=\kappa_{1}, \chi\left(\beta \beta^{\sigma}\right)=\kappa_{2}$ by applying

$$
\left(\begin{array}{ll}
0 & 1 \\
1 & 0
\end{array}\right) \in P^{-1} O_{2}^{-}(q) P, \quad\left(\begin{array}{cc|c}
0 & 1 & \mathbf{0} \\
1 & 0 & \mathbf{0} \\
\hline \mathbf{0} & \mathbf{0} & I_{n-2}
\end{array}\right) \in H_{J} .
$$

For

$$
\left(\begin{array}{ccc}
\alpha & \beta & \\
\alpha^{\sigma} & \beta^{\sigma} & \mathbf{0}
\end{array}\right),\left(\begin{array}{ccc}
\alpha^{\prime} & \beta^{\prime} & \\
\alpha^{\prime \sigma} & \beta^{\prime \sigma} & \mathbf{0}
\end{array}\right) \in \Lambda_{\mathrm{III}}^{+-}\left(\left\{D, D^{\sigma}\right\},\left\{\kappa_{1}, \kappa_{2}\right\}\right)
$$


with $\alpha \beta^{\sigma}=\alpha^{\prime} \beta^{\prime \sigma}=D, \chi\left(\alpha \alpha^{\sigma}\right)=\chi\left(\alpha^{\prime} \alpha^{\prime \sigma}\right)=\kappa_{1}, \chi\left(\beta \beta^{\sigma}\right)=\chi\left(\beta^{\prime} \beta^{\prime \sigma}\right)=\kappa_{2}$, we claim that there exist $\xi \in G F\left(q^{2}\right)^{\times}\left(\xi^{\sigma}=\xi^{-1}\right), z \in G F(q)^{\times}$such that

$$
\left(\begin{array}{cc}
\xi & 0 \\
0 & \xi^{-1}
\end{array}\right)\left(\begin{array}{cc}
\alpha & \beta \\
\alpha^{\sigma} & \beta^{\sigma}
\end{array}\right)\left(\begin{array}{cc}
z & 0 \\
0 & z^{-1}
\end{array}\right)=\left(\begin{array}{cc}
\alpha^{\prime} & \beta^{\prime} \\
\alpha^{\prime \sigma} & \beta^{\prime \sigma}
\end{array}\right),
$$

i.e. $\alpha^{\prime}=\xi z \alpha, \beta^{\prime}=\xi z^{-1} \beta$. Since $\chi\left(\alpha \alpha^{\sigma}\right)=\chi\left(\alpha^{\prime} \alpha^{\prime \sigma}\right) \neq 0$, there exists $z \in G F(q)^{\times}$ such that $\alpha^{\prime} \alpha^{\prime \sigma} / \alpha \alpha^{\sigma}=z^{2}$. Set $\xi=\alpha^{\prime} / \alpha z$. Then $\xi \xi^{\sigma}=\alpha^{\prime} \alpha^{\prime \sigma} / \alpha \alpha^{\sigma} z^{2}=1$ and $\xi z^{-1} \beta=\alpha^{\prime} \beta / \alpha z^{2}=\alpha^{\sigma} \beta / \alpha^{\prime \sigma}=\beta^{\prime}$ by $\alpha^{\sigma} \beta=\alpha^{\prime \sigma} \beta^{\prime}=D^{\sigma}$. Thus these $z, \xi$ meet the requirement. So the claim is proved and hence $P^{-1} O_{2}^{-}(q) P \times H_{J}$ is transitive on $\Lambda_{\text {III }}^{+-}\left(\left\{D, D^{\sigma}\right\},\left\{\kappa_{1}, \kappa_{2}\right\}\right)$.

Apparently, each matrix of type II belongs to exactly one of $\Omega_{\mathrm{II}}^{+-}(D, \kappa)$ and each matrix of type III belongs to exactly one of $\Omega_{\mathrm{III}}^{+-}\left(\left\{D, D^{\sigma}\right\},\left\{\kappa_{1}, \kappa_{2}\right\}\right)$. Thus the theorem is proved except for the number of orbits.

We count the number of orbits $\Omega_{\text {III }}^{+-}\left(\left\{D, D^{\sigma}\right\},\left\{\kappa_{1}, \kappa_{2}\right\}\right)$. Let $\varphi$ denote the mapping $\xi \mapsto \xi \xi^{\sigma}$ from $G F\left(q^{2}\right)^{\times}$to $G F(q)^{\times}$. $\varphi$ is surjective and $\operatorname{ker} \varphi$ is of order $q+1 .\left.\operatorname{Im} \varphi\right|_{G F(q)^{\times}}$is the set of squares of $G F(q)^{\times}$and $\left.\operatorname{ker} \varphi\right|_{G F(q)^{\times}}$is of order 2. So for a square $s$ of $G F(q)^{\times}$, there are $(q+1)-2$ elements $D \in G F\left(q^{2}\right)^{\times}-G F(q)^{\times}$ such that $D D^{\sigma}=s$, i.e. $(q-1) / 2$ such pairs $\left\{D, D^{\sigma}\right\}$. For such a pair $\left\{D, D^{\sigma}\right\}$, the choice of $\left\{\kappa_{1}, \kappa_{2}\right\}$ such that $\chi\left(D D^{\sigma}\right)=\kappa_{1} \kappa_{2}$ is either $\{1,1\}$ or $\{-1,-1\}$. Since there are $(q-1) / 2$ squares of $G F(q)^{\times}$, the number of $\left(\left\{D, D^{\sigma}\right\},\left\{\kappa_{1}, \kappa_{2}\right\}\right)$ such that $\chi\left(D D^{\sigma}\right)=\kappa_{1} \kappa_{2}=1$ is $(q-1) / 2((q-1) / 2) \times 2$. For a non-square $t \in G F(q)^{\times}$, there are $q+1$ elements $D \in G F\left(q^{2}\right)^{\times}-G F(q)^{\times}$such that $D D^{\sigma}=t$, i.e. $(q+1) / 2$ such pairs $\left\{D, D^{\sigma}\right\}$. For such a pair $\left\{D, D^{\sigma}\right\}$, the choice of $\left\{\kappa_{1}, \kappa_{2}\right\}$ such that $\chi\left(D D^{\sigma}\right)=\kappa_{1} \kappa_{2}$ is only $\{1,-1\}$. Since there are $(q-1) / 2$ non-squares of $G F(q)^{\times}$, the number of $\left(\left\{D, D^{\sigma}\right\},\left\{\kappa_{1}, \kappa_{2}\right\}\right)$ such that $\chi\left(D D^{\sigma}\right)=\kappa_{1} \kappa_{2}=-1$ is $(q-1) / 2((q+1) / 2)$. Thus the number of orbits $\Omega_{\text {III }}^{+-}\left(\left\{D, D^{\sigma}\right\},\left\{\kappa_{1}, \kappa_{2}\right\}\right)$ is

$$
\frac{q-1}{2} \frac{q-1}{2} \times 2+\frac{q-1}{2} \frac{q+1}{2}=\frac{1}{4}(q-1)(3 q-1) .
$$

Apparently, the number of orbits $\Omega_{\mathrm{I}}, \Omega_{\mathrm{II}}^{+-}(D, \kappa)$ is $1,2 q$, respectively.

3.6. The case $\left(J, J^{\prime}\right)=\left(J_{2}^{-}, J_{2}^{-}\right)$

We apply Corollary 2.3 with

$$
\begin{gathered}
J=J^{\prime}=J_{2}^{-}, \quad m^{\prime}=2, \quad O_{m^{\prime}}\left(q, J^{\prime}\right)=O_{2}^{-}(q), \\
G_{J}=U_{2}(q) \rtimes\left(H_{J} \times K_{2}(q)\right), \quad H_{J}=\left\{\left(\begin{array}{cc}
A & 0 \\
0 & I_{n-2}
\end{array}\right) \mid A \in O_{2}^{-}(q)\right\} .
\end{gathered}
$$


Recall that

$$
P=\left(\begin{array}{cc}
1 & 1 \\
1 / \sqrt{\epsilon} & -1 / \sqrt{\epsilon}
\end{array}\right), \quad\langle\sigma\rangle=\operatorname{Gal}\left(G F\left(q^{2}\right) / G F(q)\right),
$$

and $\chi$ is the quadratic residue character of $G F(q)$. Set

$$
\widetilde{P}=\left(\begin{array}{cc}
P & 0 \\
0 & I_{n-2}
\end{array}\right) \in G L_{n}\left(q^{2}\right) .
$$

Set

$$
\begin{aligned}
G F(q)^{\sim} & =\{0\} \cup G F(q)^{\times} \cup \sqrt{\epsilon} G F(q)^{\times}=\left\{\xi \in G F\left(q^{2}\right) \mid \xi^{2} \in G F(q)\right\} \\
& =\left\{\xi^{(q+1) / 2} \mid \xi \in G F\left(q^{2}\right)\right\} .
\end{aligned}
$$

Then the scalar -1 acts on $G F(q)^{\sim} \times G F(q)^{\sim}$ by $\left(D_{1}, D_{2}\right) \mapsto\left(-D_{1},-D_{2}\right)$ and $\sigma$ acts on $G F(q)^{\sim} \times G F(q)^{\sim}$ by $\left(D_{1}, D_{2}\right) \mapsto\left(D_{1}^{\sigma}, D_{2}^{\sigma}\right)$. Let $\tau$ denote the mapping $\left(D_{1}, D_{2}\right) \mapsto\left(D_{2}, D_{1}\right)$ from $G F(q)^{\sim} \times G F(q)^{\sim}$ onto itself. Let $G F(q)^{\sim} \times G F(q)^{\sim} / \sim$ denote the set of orbits of the group $\langle-1\rangle \times\langle\sigma\rangle \times\langle\tau\rangle$ on $G F(q)^{\sim} \times G F(q)^{\sim}$.

THEOREM 3.6. $\mathrm{O}_{2}\left(q, J_{2}^{-}\right) \backslash \mathcal{M}_{2, n}^{*}(q) / G_{J_{2}^{-}}$consists of:

(i) $\Omega_{\mathrm{I}}=\left\{\left(M_{0}, M_{1}\right) \mid M_{0} \in \mathcal{M}_{2,2}(q), M_{1} \in \mathcal{M}_{2, n-2}^{*}(q)\right\}$;

(ii) $\Omega_{\mathrm{II}}^{--}(D, \kappa)=P \Lambda_{\mathrm{II}}^{--}(D, \kappa) \widetilde{P}^{-1} K_{2}(q)$ for $(D, \kappa) \in G F(q)^{\times} \times\{ \pm 1\}$, where

$$
\begin{aligned}
\Lambda_{\mathrm{II}}^{--}(D, \kappa)= & \left\{\left(\begin{array}{cccc}
\alpha & \beta & \gamma & \\
\beta^{\sigma} & \alpha^{\sigma} & \gamma^{\sigma} & \mathbf{0}
\end{array}\right) \in \mathcal{M}_{2, n}^{*}\left(q^{2}\right) \mid \chi\left(\gamma \gamma^{\sigma}\right)=\kappa,\right. \\
& \left.\frac{\left(\alpha \gamma^{\sigma}-\beta^{\sigma} \gamma\right)\left(\alpha^{\sigma} \gamma-\beta \gamma^{\sigma}\right)}{\gamma \gamma^{\sigma}}=D\right\} ;
\end{aligned}
$$

(iii) $\Omega_{\mathrm{III}}^{--}\left(D_{1}, D_{2}\right)=P \Lambda_{\mathrm{III}}^{--}\left(D_{1}, D_{2}\right) \widetilde{P}^{-1}$ for $\left(D_{1}, D_{2}\right) \quad \in G F(q)^{\sim} \times$ $G F(q)^{\sim} / \sim\left(D_{1}^{2} \neq D_{2}^{2}\right)$, where

$$
\begin{aligned}
& \Lambda_{\mathrm{III}}^{--}\left(D_{1}, D_{2}\right) \\
& \quad=\left\{\left(\begin{array}{ccc}
\alpha & \beta & \\
\beta^{\sigma} & \alpha^{\sigma} & \mathbf{0}
\end{array}\right) \in \mathcal{M}_{2, n}^{*}\left(q^{2}\right) \mid\left(\alpha^{(q+1) / 2}, \beta^{(q+1) / 2}\right) \sim\left(D_{1}, D_{2}\right)\right\} .
\end{aligned}
$$

There are $1+2(q-1)+\frac{3}{4}(q-1)^{2}$ orbits. 
Proof. Let $\Omega_{\mathrm{I}}, \Omega_{\mathrm{II}}, \Omega_{\mathrm{III}}$ be the set of matrices of type I, II, III, respectively, as defined in the proof of Theorem 3.5. They are fixed as a set by the action of $O_{2}^{-}(q), G_{J}$ $\left(J=J_{2}^{-}\right)$, and $\Omega_{\mathrm{I}}$ is an orbit of $O_{2}^{-}(q) \times G_{J}$ as before.

The action of $O_{2}^{-}(q) \times G_{J}$ on $\mathcal{M}_{2, n}^{*}(q)$ is isomorphic to that of $\left(P^{-1} O_{2}^{-}(q) P\right) \times$ $\left(\widetilde{P}^{-1} G_{J} \widetilde{P}\right)$ on $P^{-1} \mathcal{M}_{2, n}^{*}(q) \widetilde{P}$ by the correspondence $(A \times B, M) \mapsto\left(P^{-1} A P \times\right.$ $\left.\widetilde{P}^{-1} B \widetilde{P}, P^{-1} M \widetilde{P}\right)$ from $\left(O_{2}^{-}(q) \times G_{J}\right) \times \mathcal{M}_{2, n}^{*}(q)$ to $\left(\left(P^{-1} O_{2}^{-}(q) P\right) \times\right.$ $\left.\left(\widetilde{P}^{-1} G_{J} \widetilde{P}\right)\right) \times P^{-1} \mathcal{M}_{2, n}^{*}(q) \widetilde{P}$. Note that $\widetilde{P}^{-1} G_{J} \widetilde{P}=U_{2}^{\prime}(q) \rtimes\left(H_{J}^{\prime} \times K_{2}(q)\right)$, where, by Lemma 2.4 (ii),

$$
\begin{gathered}
U_{2}^{\prime}(q)=\left\{\left(\begin{array}{cc}
I_{2} & 0 \\
B & I_{n-2}
\end{array}\right) \mid B=\left(\begin{array}{cc}
\lambda_{1} & \lambda_{1}^{\sigma} \\
\vdots & \vdots \\
\lambda_{n-2} & \lambda_{n-2}^{\sigma}
\end{array}\right)\left(\lambda_{i} \in G F\left(q^{2}\right)\right)\right\}, \\
H_{J}^{\prime}=\left\{\left(\begin{array}{cc}
A & 0 \\
0 & I_{n-2}
\end{array}\right) \mid A \in P^{-1} O_{2}^{-}(q) P\right\}, \\
P^{-1} O_{2}^{-}(q) P=\left\langle\left(\begin{array}{cc}
\xi & 0 \\
0 & \xi^{-1}
\end{array}\right),\left(\begin{array}{ll}
0 & 1 \\
1 & 0
\end{array}\right) \mid \xi \in G F\left(q^{2}\right)^{\times}, \xi^{\sigma}=\xi^{-1}\right\rangle .
\end{gathered}
$$

Also, note that $\widetilde{P}$ commutes with each element of $K_{2}(q)$. It can be easily checked that each of $\Lambda_{\mathrm{II}}^{--}(D, \kappa) K_{2}(q), \Lambda_{\mathrm{III}}^{--}\left(D_{1}, D_{2}\right)$ is fixed as a set by the action of $P^{-1} O_{2}^{-}(q) P, \stackrel{P}{P}^{-1} G_{J} \widetilde{P}=U_{2}^{\prime}(q) \rtimes\left(H_{J}^{\prime} \times K_{2}(q)\right)$. So each of $\Omega_{\mathrm{II}}^{--}(D, \kappa)$, $\Omega_{\text {III }}^{--}\left(D_{1}, D_{2}\right)$ is fixed as a set by the action of $O_{2}^{-}(q), G_{J}$. So to show $\Omega_{\mathrm{II}}^{--}(D, \kappa)$ (respectively $\Omega_{\mathrm{III}}^{--}\left(D_{1}, D_{2}\right)$ ) is an orbit of $O_{2}^{-}(q) \times G_{J}$, it suffices to show that $\left(P^{-1} O_{2}^{-}(q) P\right) \times\left(U_{2}^{\prime}(q) H_{J}^{\prime}\right)$ is transitive on $\Lambda_{\text {II }}^{--}(D, \kappa)\left(\right.$ respectively $\left.\Lambda_{\text {III }}^{--}\left(D_{1}, D_{2}\right)\right)$.

As in the proof of Theorem 3.5, fix $\gamma_{0} \in G F\left(q^{2}\right)^{\times}$such that $\chi\left(\gamma_{0} \gamma_{0}^{\sigma}\right)=\kappa$. Then every element of $\Lambda_{\mathrm{II}}^{--}(D, \kappa)$ is sent to $\left(\begin{array}{ccc}\alpha & \beta & \gamma_{0} \\ \beta^{\sigma} & \alpha^{\sigma} & \gamma_{0}^{\sigma}\end{array}\right)$ for some $\alpha, \beta \in G F\left(q^{2}\right)^{\times}$ by the action of $P^{-1} O_{2}^{-}(q) P$ and $K_{2}(q)$. Fix $\zeta_{0} \in G F\left(q^{2}\right)^{\times}$such that $\zeta_{0} \zeta_{0}^{\sigma}=$ $D / \gamma_{0} \gamma_{0}^{\sigma}$. Then we have

$$
\frac{\alpha}{\gamma_{0}}-\left(\frac{\beta}{\gamma_{0}}\right)^{\sigma}=\xi \zeta_{0}
$$

for some $\xi \in G F\left(q^{2}\right)^{\times}$such that $\xi^{\sigma}=\xi^{-1}$, since

$$
\left(\frac{\alpha}{\gamma_{0}}-\left(\frac{\beta}{\gamma_{0}}\right)^{\sigma}\right)\left(\left(\frac{\alpha}{\gamma_{0}}\right)^{\sigma}-\frac{\beta}{\gamma_{0}}\right)=D / \gamma_{0} \gamma_{0}^{\sigma}
$$

and the mapping $\zeta \mapsto \zeta \zeta^{\sigma}$ from $G F\left(q^{2}\right)^{\times}$to $G F(q)^{\times}$is surjective and has the kernel $\left\{\xi \in G F(q)^{\times} \mid \xi^{\sigma}=\xi^{-1}\right\}$. By the action of $H_{J}^{\prime}$, we may assume that $\xi=1$. 
Fix $\alpha_{0}, \beta_{0} \in G F\left(q^{2}\right)$ such that

$$
\frac{\alpha_{0}}{\gamma_{0}}-\left(\frac{\beta_{0}}{\gamma_{0}}\right)^{\sigma}=\zeta_{0}
$$

Given $\alpha, \beta \in G F\left(q^{2}\right)$ such that

$$
\frac{\alpha}{\gamma_{0}}-\left(\frac{\beta}{\gamma_{0}}\right)^{\sigma}=\zeta_{0},
$$

set

$$
\lambda=\left(\frac{\beta}{\gamma_{0}}\right)^{\sigma}-\left(\frac{\beta_{0}}{\gamma_{0}}\right)^{\sigma} .
$$

Then $\alpha=\alpha_{0}+\lambda \gamma_{0}, \beta=\beta_{0}+\lambda^{\sigma} \gamma_{0}$, i.e.

$$
\left(\begin{array}{ccc}
\alpha & \beta & \gamma_{0} \\
\beta^{\sigma} & \alpha^{\sigma} & \gamma_{0}^{\sigma}
\end{array}\right)=\left(\begin{array}{ccc}
\alpha_{0} & \beta_{0} & \gamma_{0} \\
\beta_{0}^{\sigma} & \alpha_{0}^{\sigma} & \gamma_{0}^{\sigma}
\end{array}\right)\left(\begin{array}{ccc}
1 & 0 & 0 \\
0 & 1 & 0 \\
\lambda & \lambda^{\sigma} & 1
\end{array}\right)
$$

This means that $\left(\begin{array}{cccc}\alpha & \beta & \gamma_{0} & 0 \\ \beta^{\sigma} & \alpha^{\sigma} & \gamma_{0}^{\sigma} & \mathbf{0}\end{array}\right)$ is sent to $\left(\begin{array}{cccc}\alpha_{0} & \beta_{0} & \gamma_{0} & 0 \\ \beta_{0}^{\sigma} & \alpha_{0}^{\sigma} & \gamma_{0}^{\sigma} & 0\end{array}\right)$ by $U_{2}^{\prime}(q)$. Therefore $\left(P^{-1} O_{2}^{-}(q) P\right) \times\left(U_{2}^{\prime}(q) H_{J}^{\prime}\right)$ is transitive on $\Lambda_{\mathrm{II}}^{-}(D, \kappa) K_{2}(q)$.

Next, we want to show that $\left(P^{-1} O_{2}^{-}(q) P\right) \times H_{J}^{\prime}$ is transitive on $\Lambda_{\text {III }}^{--}\left(D_{1}, D_{2}\right)$. For

$$
\left(\begin{array}{ccc}
\alpha & \beta & \\
\beta^{\sigma} & \alpha^{\sigma} & \mathbf{0}
\end{array}\right) \in \Lambda_{\mathrm{III}}^{--}\left(D_{1}, D_{2}\right)
$$

we may assume that $\alpha^{(q+1) / 2}=D_{1}, \beta^{(q+1) / 2}=D_{2}$ by the action of

$$
\left(\begin{array}{ll}
0 & 1 \\
1 & 0
\end{array}\right),\left(\begin{array}{cc}
\xi & 0 \\
0 & \xi^{-1}
\end{array}\right) \in P^{-1} O_{2}^{-}(q) P\left(\xi^{(q+1) / 2}=-1\right), \quad\left(\begin{array}{cc|c}
0 & 1 & \mathbf{0} \\
1 & 0 & \mathbf{0} \\
\hline \mathbf{0} & \mathbf{0} & I_{n-2}
\end{array}\right) \in H_{J}^{\prime}
$$

For

$$
\left(\begin{array}{ccc}
\alpha & \beta & \\
\beta^{\sigma} & \alpha^{\sigma} & \mathbf{0}
\end{array}\right),\left(\begin{array}{ccc}
\alpha^{\prime} & \beta^{\prime} & \\
\beta^{\prime \sigma} & \alpha^{\prime \sigma} & \mathbf{0}
\end{array}\right) \in \Lambda_{\mathrm{III}}^{--}\left(D_{1}, D_{2}\right)
$$

with $\alpha^{(q+1) / 2}=\alpha^{\prime(q+1) / 2}=D_{1}, \beta^{(q+1) / 2}=\beta^{\prime(q+1) / 2}=D_{2}$, we claim that there exist $\xi_{1}, \xi_{2} \in G F\left(q^{2}\right)^{\times}\left(\xi_{1}^{\sigma}=\xi_{1}^{-1}, \xi_{2}^{\sigma}=\xi_{2}^{-1}\right)$ such that

$$
\left(\begin{array}{cc}
\xi_{1} & 0 \\
0 & \xi_{1}^{-1}
\end{array}\right)\left(\begin{array}{cc}
\alpha & \beta \\
\beta^{\sigma} & \alpha^{\sigma}
\end{array}\right)\left(\begin{array}{cc}
\xi_{2} & 0 \\
0 & \xi_{2}^{-1}
\end{array}\right)=\left(\begin{array}{cc}
\alpha^{\prime} & \beta^{\prime} \\
\beta^{\prime \sigma} & \alpha^{\prime \sigma}
\end{array}\right),
$$

i.e. $\alpha^{\prime}=\xi_{1} \xi_{2} \alpha, \beta^{\prime}=\xi_{1} \xi_{2}^{-1} \beta$. 
Suppose that $D_{1} \neq 0, D_{2} \neq 0$. Since $\left(\alpha^{\prime} / \alpha\right)^{(q+1) / 2}=1, \alpha^{\prime} / \alpha$ is contained in the cyclic subgroup of order $(q+1) / 2$ of $G F\left(q^{2}\right)^{\times}$and so there exists $\lambda \in G F\left(q^{2}\right)^{\times}$ such that $\lambda^{q+1}=1, \lambda^{2}=\alpha^{\prime} / \alpha$. Since $\left(\beta^{\prime} / \beta\right)^{(q+1) / 2}=1$, there exists $\mu \in G F\left(q^{2}\right)^{\times}$ such that $\mu^{q+1}=1, \mu^{2}=\beta^{\prime} / \beta$. Set $\xi_{1}=\lambda \mu$ and $\xi_{2}=\lambda / \mu$. Then $\xi_{1} \xi_{2} \alpha=\lambda^{2} \alpha=$ $\alpha^{\prime}, \xi_{1} \xi_{2}^{-1} \beta=\mu^{2} \beta=\beta^{\prime}$ and so $\xi_{1}, \xi_{2}$ meet the requirement.

Suppose that $D_{1}=0$. Then $D_{2} \neq 0$, since $D_{1}^{2} \neq D_{2}^{2}$. Set $\xi_{1}=\beta^{\prime} / \beta, \xi_{2}=1$. Then $\xi_{1}^{q+1}=1$ and so $\xi_{1}, \xi_{2}$ meet the requirement.

Suppose that $D_{2}=0$. Then $D_{1} \neq 0$ and $\xi_{1}=\alpha^{\prime} / \alpha, \xi_{2}=1$ meet the requirement. Thus the claim is proved and hence $P^{-1} O_{2}^{-}(q) P \times H_{J}^{\prime}$ is transitive on $\Lambda_{\mathrm{III}}^{--}\left(D_{1}, D_{2}\right)$.

By Lemma 2.4, each matrix of type II belongs to exactly one of $\Omega_{\mathrm{II}}^{--}(D, \kappa)$ and each matrix of type III belongs to exactly one of $\Omega_{\text {III }}^{--}\left(D_{1}, D_{2}\right)$. Thus the theorem is proved except for the number of orbits.

We count the number of orbits $\Omega_{\text {III }}^{--}\left(D_{1}, D_{2}\right)$. The length of the orbit of $\langle-1\rangle \times\langle\sigma\rangle \times\langle\tau\rangle$ containing $\left(D_{1}, D_{2}\right)$ with $D_{1}^{2} \neq D_{2}^{2}$ is four if $\left(D_{1}, D_{2}\right) \in$ $\left(G F(q)^{\times} \times G F(q)^{\times}\right) \cup\left(\sqrt{\epsilon} G F(q)^{\times} \times \sqrt{\epsilon} G F(q)^{\times}\right)$, eight if $\left(D_{1}, D_{2}\right) \in\left(G F(q)^{\times} \times\right.$ $\left.\sqrt{\epsilon} G F(q)^{\times}\right) \cup\left(\sqrt{\epsilon} G F(q)^{\times} \times G F(q)^{\times}\right)$and four if $D_{1}=0$ or $D_{2}=0$. There are $(q-1)(q-3)+(q-1)(q-3)$ many $\left(D_{1}, D_{2}\right)$ in $\left(G F(q)^{\times} \times G F(q)^{\times}\right) \cup\left(\sqrt{\epsilon} G F(q)^{\times} \times\right.$ $\left.\sqrt{\epsilon} G F(q)^{\times}\right)$such that $D_{1}^{2} \neq D_{2}^{2}$. There are $(q-1)^{2}+(q-1)^{2}$ many $\left(D_{1}, D_{2}\right)$ in $\left(G F(q)^{\times} \times \sqrt{\epsilon} G F(q)^{\times}\right) \cup\left(\sqrt{\epsilon} G F(q)^{\times} \times G F(q)^{\times}\right)$such that $D_{1}^{2} \neq D_{2}^{2}$. There are $2(q-1)+2(q-1)$ many $\left(D_{1}, D_{2}\right)$ such that $D_{1}^{2} \neq D_{2}^{2}$ and $D_{1} D_{2}=0$. Thus the number of orbits $\Omega_{\mathrm{III}}^{--}\left(D_{1}, D_{2}\right)$ is $\frac{1}{2}(q-1)(q-3)+\frac{1}{4}(q-1)^{2}+(q-1)=\frac{3}{4}(q-1)^{2}$. Apparently, the number of orbits $\Omega_{\mathrm{I}}, \Omega_{\mathrm{II}}^{--}(D, \kappa)$ is $1,2(q-1)$, respectively.

\section{REFERENCES}

[1] E. Bannai and T. Ito. Algebraic Combinatorics I. Benjamin/Cummings, Menlo Park, CA, 1984.

[2] D. G. Higman. Coherent configurations, Part I: ordinary representation theory. Geom. Dedicata 4 (1975), 1-32.

[3] S. Hobart, and T. Ito. The structure of nonthin irreducible $T$-modules of endpoint 1: ladder bases and classical parameters. J. Algebraic Combin. 7 (1988), 53-75.

[4] P. Terwilliger. The subconstituent algebra of a distance-regular graph; thin modules with endpoint one. Linear Algebra Appl. 356 (2002), 157-187.

Seiichi Iwamoto

Graduate School of Natural Science and Technology

Kanazawa University

Kakuma-machi

Kanazawa 920-1192

Japan

(E-mail: iwamoto@kappa.s.kanazawa-u.ac.jp) 